\title{
El Ecosistema Emprendedor en las Principales Instituciones de Enseñanza e Investigación Agrícola de México
}

\author{
Jimena Achiquen Millán ${ }^{1}$, Vinicio Horacio Santoyo Cortés ${ }^{*}$, Enrique Genaro Martínez González ${ }^{1}$ \\ Manrrubio Muñoz Rodríguez ${ }^{1}$
}

Resumen: Este estudio analiza el emprendimiento asociado a las dos universidades agropecuarias más importantes de México para reconocer a los actores que interaccionan en este proceso a fin de proponer acciones que potencialicen la transferencia de conocimiento de la academia hacia el sector productivo. A través de un estudio cualitativo se identificó la estructura y funciones de los actores involucrados en el ecosistema emprendedor de estas instituciones. Se concluye que las universidades estudiadas generan conocimientos y desarrollos tecnológicos con posibilidad de utilidad comercial. No obstante, la mayoría de ellos no son llevados al mercado debido a que su promoción queda a cargo de los emprendedores, de forma que los resultados del ecosistema analizado son más bien circunstanciales y no son resultado de una estrategia bien definida. Por ello, se proponen cambios en los roles en las estructuras de las instituciones que les permitan evolucionar hacia universidades emprendedoras.

Palabras clave: ecosistema emprendedor universitario; universidad emprendedora; emprendimientos universitarios; emprendimiento académico

\begin{abstract}
The entrepreneurial ecosystem in the main agricultural universities in Mexico
This study analyzes the entrepreneurship associated with the two most important agricultural universities in Mexico to recognize the actors that interact in this process in order to propose actions that enhance the transfer of knowledge from the academy to the productive sector. Through a qualitative study, the structure and functions of the actors involved in the entrepreneurial ecosystem of these institutions were identified. It is concluded that the universities studied generate knowledge and technological developments with the possibility of commercial utility. However, most of them are not commercialized because their promotion is left to the responsibility of entrepreneurs, so that the results of the analyzed ecosystem are circumstantial and are not the result of a well-defined strategy. For this reason, changes are proposed in the roles in the structures of the institutions that allow them to develop towards entrepreneurial universities.
\end{abstract}

Keywords: entrepreneurial university ecosystem; entrepreneurial university; university ventures; academic entrepreneurship

Submitted: July $26^{\text {th }}, 2021 /$ Approved: October $26^{\text {th }}, 2021$

\section{Introducción}

Existe cada vez mayor convencimiento en los gobiernos que la tecnología y los sectores basados en el conocimiento son una fuente de oportunidades de empleo, por lo que en muchos países las universidades y centros de investigación se consideran como una parte integral de instituciones públicas que actúan como centros de atracción para individuos y empresas contribuyendo a la mejora de la industria local (Huang-Saad, Fay, \& Sheridan, 2017).

Es por ello que, tanto las autoridades académicas como las públicas están prestando cada vez más atención a la transferencia de tecnología de las universidades a través de la creación de empresas (Roberts \& Malone, 1996; Schillo, 2018).

Por otra parte, la investigación sobre el espíritu empresarial de industrias emergentes en países en desarrollo es relativamente escasa; sin embargo, resulta importante su estudio considerando que en estos contextos las empresas se enfrentan a mayores limitaciones de recursos y a mercados inmaduros debido a su pequeñez y novedad, lo cual genera diferencias en el comportamiento empresarial con respecto a países desarrollados (Cai, Peng, \& Wang, 2018; Hisrich, Ge, Gao, \& Sheng, 2016; OCDE, 2012).

Por lo anterior, el objetivo de esta investigación fue analizar el emprendimiento académico asociado a las Instituciones de Enseñanza e Investigación Agropecuaria (IEIA) en México, el Colegio de Postgraduados (COLPOS) y la Universidad Autónoma Chapingo (UACh); a través de la identificación estructural y funcional de los actores que interaccionan en este proceso a fin de proponer acciones que sirvan para potencializar la transferencia de conocimiento de la academia hacia el sector productivo.

\section{Emprendimiento académico}

En el sentido más amplio, el emprendimiento es el acto de creación organizacional, renovación o innovación que ocurre dentro o fuera de una organización existente (Sharma \& Chrisman, 1999). Por tanto, el emprendedor es un actor capaz de descubrir oportunidades, de apreciarlas en su justo valor y de reunir y organizar los recursos necesarios para aprovechar dichas oportunidades (Meredith, Nelson, \& Neck, 1986).

(1) Universidad Autónoma Chapingo. CIESTAAM.

*Autor de correspondencia: hsantoyo@ciestaam.edu.mx 
El fenómeno de emprendimiento dentro de las universidades es reciente y por consiguiente la investigación sobre cómo las universidades están tratando de promover la creación de empresas es aún incipiente (Muscio \& Ramaciotti, 2019; Ndonzuau, Pirnay, \& Surlemont, 2002). Diversos autores (Abreu, Demirel, Grinevich, \& KarataşÖzkan, 2016; Etzkowitz, Webster, Gebhardt, \& Cantisano Terra, 2000; Guerrero, Urbano, Fayolle, Klofsten, \& Mian, 2016; Kalar \& Antoncic, 2015) mencionan que, además de la docencia y la investigación, las universidades están implementando una tercera misión, la cual implica el intercambio de conocimientos en su sentido más amplio, incluida la comercialización de la investigación, las asociaciones entre la universidad y la industria y todos los compromisos empresariales relacionados.

Clark (1998) utiliza el término emprendimiento en el ámbito universitario como una característica de las universidades y sus departamentos que generan cambios organizacionales con matices empresariales para transformarse en universidades emprendedoras, tales actividades se llevan a cabo con el objetivo de mejorar el desempeño económico, así como obtener ingresos económicos (Etzkowitz et al., 2000).

Por su parte, Anderseck (2004) hace referencia a dos orientaciones en la formación y cualificación de los emprendedores académicos. Una de ellas lo asocia al aspecto académico y se define por la enseñanza de asignaturas teóricas alineadas al uso práctico, mientras que la otra orientación está dirigida en ofrecer experiencias prácticas a empresarios potenciales y en ayudar a las empresas emergentes.

En las últimas décadas la creación de empresas derivadas de las universidades y centros de investigación ha ido ganando importancia al considerarse como un medio eficaz de transferencia de tecnología que lleva a la creación de empleo y riqueza (Klofsten \& Jones-Evans, 2000; Rodeiro Pazos, Fernández López, Rodríguez Sandiás, \& Otero González, 2010; Siegel, Waldman, Atwater, \& Link, 2003).

Actualmente no existe un consenso sobre el término para aludir a estas empresas, ya que uno se usa de manera diferente por varios autores, mientras que otros usan términos diferentes para describir el mismo fenómeno. En la literatura se pueden encontrar referidas como spin-off, start-ups, spin-outs (Carayannis, Rogers, Kurihara, \& Allbritton, 1998); Empresas de Base Tecnológica (EBT) (Merritt, 2012; Shearman \& Burrell, 1988) y son definidas como pequeñas empresas asociadas a industrias emergentes con vínculos universitarios claros, fuertes y activos, pues surgen de una Organización Matriz (OM) como ideas de negocios innovadoras.

En este sentido, la importancia de las EBT radica en que son un medio de transferencia de tecnología y conocimientos generados en universidades o centros de investigación y que contribuyen a la creación de empleo y riqueza, resaltando el papel de la universidad en el desarrollo económico (Carayannis et al., 1998; Klofsten \& Jones-Evans, 2000; Steffensen, Rogers, \& Speakman, 2000).

Por otro lado, varios autores (Aportela Rodríguez \& Gallego Gómez, 2015; Klofsten \& Jones-Evans, 2000; Steffensen et al., 2000) apuntan a que las relaciones entre una EBT y su respectiva OM pueden ser de beneficio mutuo para ambas entidades, por mencionar algunas: asesoría legal y financiera, introducción de los emprendedores en redes empresariales concretas u ofrecer espacios de trabajo para que la empresa se pueda desarrollar en su etapa inicial. Ecosistema emprendedor universitario

\section{Ecosistema emprendedor universitario}

Las EBT están involucradas dinámicamente en el proceso de difusión del conocimiento e incorporadas en el ecosistema empresarial (Prencipe, Corsi, Rodríguez-Gulías, Fernández-López, \& RodeiroPazos, 2020), por lo que resulta importante contextualizar el funcionamiento de estas empresas en el entorno donde se desenvuelven. Se han definido a estos ecosistemas como un conjunto de factores interdependientes y coordinados que tienen como objetivo permitir el espíritu empresarial, siendo las universidades quienes organizan el flujo de conocimiento para permitir la creación de estos ecosistemas (Audretsch, Cunningham, Kuratko, Lehmann, \& Menter, 2019).

Así, los ecosistemas empresariales universitarios se basan en combinaciones culturales, financieras, de factores humanos, institucionales y políticas dentro de una región, con el objetivo de apoyar el desarrollo y crecimiento de EBT, incentivando a emprendedores y a otros actores para iniciar, financiar y apoyar a empresas de alto riesgo (Lahikainen, Kolhinen, Ruskovaara, \& Pihkala, 2019). El enfoque de ecosistema emprendedor resulta importante para comprender las relaciones entre el proceso de emprendimiento y su entorno local y son una herramienta de política para ayudar a catalizar una economía sostenible y dirigida por el espíritu emprendedor (Spigel \& Harrison, 2017).

Por su parte, Prencipe et al. (2020) demostraron que el contexto regional explica el crecimiento en términos de empleo y de ventas en empresas universitarias españolas, pero sugieren que las condiciones pueden no tener un efecto automático en el crecimiento de la empresa, a menos que se diseñen e implementen políticas orientadas al desarrollo de infraestructura, financiamiento y redes de apoyo para que sirvan en la creación de empresas exitosas y el crecimiento económico posterior.

Lahikainen et al. (2019), también reconocen que el desarrollo de las universidades emprendedoras está condicionado por factores externos formales e informales e internos relacionados con los recursos y capacidades. Los factores externos incluyen la organización empresarial y el gobierno, nuevos métodos de enseñanza, incentivos, la formación de alianzas estratégicas con partes interesadas externas, oficinas de transferencia de tecnología e incubadoras de empresas.

Así, la sociedad está desafiando el modelo universitario tradicional y que el papel emergente de una universidad emprendedora debe verse de forma dual: por una parte enfocando a la investigación y desarrollo, y por otra al espíritu empresarial, contribuyendo así con la competitividad y el crecimiento económico (Guerrero et al., 2016). 
Por lo tanto, las universidades están cambiando (en contenido, estructura, gobernanza y estrategias) y evolucionando en una serie de transiciones en las que múltiples partes interesadas están continuamente moldeando y adaptando el modelo universitario (Miller, Mcadam, \& Mcadam, 2014).

En este sentido, un ecosistema emprendedor universitario podría entenderse como un conjunto de actores interconectados (potenciales y existentes), organizaciones empresariales, organizaciones de investigación y sistema político que se unen formal e informalmente mediante las iniciativas gubernamentales orientadas al desempeño del entorno empresarial (Mason \& Brown, 2014; Znagui \& Rahmouni, 2019). Isenberg (2010) identificó que un ecosistema emprendedor exitoso comprende elementos como liderazgo, cultura, mercados de capitales y clientes de mente abierta que se combinan en formas complejas, de manera que deben ser gestionados de manera integral.

\section{Materiales y métodos}

Esta investigación exploratoria es de carácter cualitativo y buscó la multiplicidad de observaciones en diversas fuentes de información. Para tal efecto, se delimitó como objeto de estudio a dos grupos de actores. El primero de ellos consideró a dos de las principales IEIA de América Latina, el COLPOS y la UACh (América Economía, 2021; QS Quacquarelli Symonds, 2021). Estas universidades se ubican a unos cuantos kilómetros de la Ciudad de México y mantienen gran influencia en su entorno, observando en los últimos años el surgimiento y desarrollo de empresas cuyos fundadores forman o formaron parte de ellas y mantienen vínculos de colaboración. Ambas, se han posicionado como instituciones líderes en México en educación pública especializada en ciencias agropecuarias (Universidad Nacional Autónoma de México, 2020). En 2018, el COLPOS tuvo una matrícula de 1,034 alumnos en 36 planes de estudio; mientras que en la UACh se prepararon 5,886 estudiantes inscritos en 53 programas académicos. En 2017, egresaron 236 y 1,363 profesionistas de educación superior y posgrado del COLPOS y de la UACh, respectivamente.

Se realizó una revisión documental de los estatutos y normatividad de cada IEIA para conocer información relacionada con la oferta formativa y su grado de especialización, así como la regulación de sus relaciones con el sector productivo. También se realizaron entrevistas semiestructuradas a representantes de áreas involucradas en la transferencia de tecnología a fin de obtener diferentes perspectivas. Estas entrevistas se basaron en las recomendaciones de Cáceres Carrasco \& Aceytuno (2015) y se obtuvo información concerniente al perfil de la IEIA, los recursos de los que dispone y los modelos de apoyo a la creación y desarrollo de empresas.

El segundo grupo de actores que se analizó fueron los emprendimientos asociados a estas dos instituciones. Ante la inexistencia de un registro oficial, se identificó a informantes clave dentro de las IEIA a fin de conformar un padrón de emprendimientos derivados de éstas.
Los emprendimientos identificados se clasificaron de acuerdo con Vitón, Castillo y Lopes Teixeira (2019), se consideró pertinente el uso de esta clasificación pues es específica para el sector de interés y está establecida en torno a la región latinoamericana.

Posteriormente, se determinó la diversidad de estos emprendimientos a través del índice de Shannon (H) (Hacker, Klaesson, Pettersson, \& Sjölander, 2013; Shannon, 1948; Warf \& Vincent, 2007). Este índice cuantifica la diversidad en función del número de categorías y su distribución proporcional, comúnmente denominada riqueza y uniformidad, respectivamente. El valor máximo del índice de Shannon depende de cada muestra; en este caso, se alcanzaría cuando los desarrollos se distribuyeran de manera uniforme en cada categoría (2.20), mientras que el valor mínimo (cero) se obtendría si todos los desarrollos se concentraran en una sola categoría. Este índice se calcula como:

$$
\mathrm{H}=-\sum \mathrm{p}_{\mathrm{i}} * \ln \mathrm{p}_{\mathrm{i}}
$$

en donde $p$ es la proporción de desarrollos en la categoría $i$.

Después, se realizaron entrevistas semiestructuradas a una muestra dirigida de casos tipo $(n=20)$; para lo cual se contactó por teléfono y correo electrónico a los emprendedores-investigadores del padrón $(\mathrm{N}=71)$ y conforme se obtuvieron las primeras entrevistas, se buscó diversificar la muestra para tener información de todas las áreas de investigación en las IEIA.

Así, se entrevistaron a 20 emprendedores-investigadores sobre las motivaciones de los emprendedores para crear una empresa, la genealogía del emprendimiento, las características técnicas y financieras de la empresa y los vínculos que mantienen con su correspondiente IEIA y con otras entidades.

Finalmente, a partir de información recabada de las IEIA y de los emprendimientos asociados, se identificaron los elementos que interactúan en el ecosistema emprendedor universitario: emprendimientos, OM, políticas públicas, capital humano, financiamiento y mercado (Audretsch \& Belitski, 2017; Isenberg, 2010; Nicotra, Romano, Del Giudice, \& Schillaci, 2018; Znagui \& Rahmouni, 2019). Resultados y discusión

\section{Resultados y discusión}

Los resultados se abordan en tres apartados: en el primero se exponen las tres modalidades de actividades empresariales identificadas en torno a las IEIA; posteriormente, se analizan los actores y los vínculos que interactúan en el ecosistema emprendedor universitario y, finalmente se discuten los retos y oportunidades que se identifican para potencializar al ecosistema emprendedor en las principales IEIA de México. 


\section{Emprendimientos derivados de instituciones de enseñanza e investigación agrícola}

Dado que en las IEIA estudiadas no existe una oficina formal sobre emprendimiento que tuviera un registro de empresas derivadas de éstas, se conformó un padrón de 71 emprendedores- investigadores a partir de información de actores clave, cuyo propósito fue reconocer la diversidad y caracterizar las modalidades de actividades empresariales en las IEIA.

Se buscó que este registro incluyera a los emprendimientos en funcionamiento relacionados con el sector agropecuario y que estuvieran fundados por profesores, investigadores, trabajadores, alumnos o egresados de las IEIA. En esta lista también se incluyó a los investigadores que desarrollaron algún tipo de propiedad intelectual registrada en la oficina de patentes y registro de variedades de su IEIA. De esta forma, los investigadores incluidos en este padrón son los más referenciados y con mayor reconocimiento en el ámbito académico.

Se constató que existe al menos un desarrollo en las nueve áreas de innovación tecnológica propuestas por Vitón et al. (2019) (Cuadro 1). A pesar de que existe mayor proporción de ellos centrados en genética y protección de cultivos y animales, el índice de Shannon = $1.60(>1)$ indica que existe diversidad en estos desarrollos (Warf \& Vincent, 2007). Hasta cierto punto este resultado es razonable, pues existen áreas de investigación relativamente nuevas en las cuales se comienzan a mostrar iniciativas empresariales.

Cuadro 1: Emprendimientos por área de innovación tecnológica

\begin{tabular}{lc}
\hline Área de innovación tecnológica & Número de emprendimientos \\
\hline Genética y protección de cultivos y animales & 33 \\
Mecanización y automatización de labores & 16 \\
Software de gestión de información y educación al productor agropecuario & 7 \\
Bioenergías y biomateriales & 4 \\
Productos y servicios alimentarios innovadores & 4 \\
Tecnologías en el procesamiento, logística y distribución de alimentos & 3 \\
Nuevos sistemas de producción & 2 \\
Big data y agricultura de precisión & 1 \\
Plataformas innovadoras de compraventa, servicios tercerizados y financiamiento & 1 \\
\hline
\end{tabular}

Fuente: Elaboración propia con base a las áreas de innovación tecnológica propuestas por Vitón et al. (2019).

A partir de entrevistas a profundidad aplicadas a una muestra de este padrón $(n=20)$ se reconocieron tres modalidades de actividades empresariales, de acuerdo con las acciones que han realizado para vincularse con la IEIA y con el mercado (Figura 1).

Figura 1: Caracterización general de modalidades empresariales dentro de las IEIA.

\begin{tabular}{|c|c|c|c|c|c|c|}
\hline \multirow{6}{*}{ 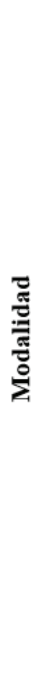 } & \multirow[b]{3}{*}{$\begin{array}{l}\text { Conocimientos con } \\
\text { potencial de } \\
\text { aprovechamiento } \\
\text { comercial }\end{array}$} & \multicolumn{5}{|c|}{ Característica } \\
\hline & & $\begin{array}{c}\text { Generación de } \\
\text { conocimientos } \\
\text { dentro de la IEIA }\end{array}$ & $\begin{array}{c}\text { Vinculación } \\
\text { emprendedor-IEIA }\end{array}$ & $\begin{array}{l}\text { Uso comercial de los } \\
\text { conocimientos } \\
\text { generados }\end{array}$ & $\begin{array}{l}\text { Formalización del } \\
\text { emprendimiento }\end{array}$ & $\begin{array}{l}\text { Vinculación con } \\
\text { el mercado }\end{array}$ \\
\hline & & $\begin{array}{l}\text { Sí, por lo que la } \\
\text { IEIA se considera } \\
\text { también como OM }\end{array}$ & $\begin{array}{l}\text { Sí, como profesores- } \\
\text { investigadores }\end{array}$ & Generalmente no & No & Esporádicamente \\
\hline & $\begin{array}{l}\text { Empresas } \\
\text { vinculadas }\end{array}$ & Generalmente no & $\begin{array}{l}\text { Sí, generalmente } \\
\text { como profesores- } \\
\text { investigadores }\end{array}$ & No aplica & $\begin{array}{l}\text { Sí, en la mayoría } \\
\text { de los casos }\end{array}$ & Sí \\
\hline & $\begin{array}{l}\text { Empresas de base } \\
\text { tecnológica }\end{array}$ & $\begin{array}{l}\text { Sí, por lo que la } \\
\text { IEIA se considera } \\
\text { también como OM }\end{array}$ & $\begin{array}{l}\text { Sí, generalmente } \\
\text { como profesores- } \\
\text { investigadores }\end{array}$ & Sí & $\begin{array}{l}\text { Sí, en la mayoría } \\
\text { de los casos }\end{array}$ & Sí \\
\hline & & $\begin{array}{l}\text { Relación con la } \\
\text { IEIA }\end{array}$ & & & & $\begin{array}{l}\text { Relación con el } \\
\text { mercado }\end{array}$ \\
\hline
\end{tabular}

Fuente: elaboración propia con información de entrevistas a emprendedores. 
El primer grupo denominado conocimientos con potencial de aprovechamiento comercial (CP) no son propiamente emprendimientos, pues en ellos el investigador no ha puesto en funcionamiento una empresa relacionada con las investigaciones que realiza. Sin embargo, se han considerado en este estudio porque pueden dar origen a los otros tipos de emprendimientos, ser la base para el diseño e implementación de políticas de apoyo al emprendimiento en las IEIA o por el reconocimiento que tienen dentro de la comunidad académica, como elemento de vinculación con el sector productivo.

Los otros dos grupos, denominados empresas vinculadas (EV) y empresas de base tecnológica (EBT) se refieren a empresas formalizadas que operan generalmente bajo una figura legal y que mantienen relación con la IEIA. Las características específicas de cada modalidad se describen en los apartados siguientes.

\section{Conocimientos con potencial de aprovechamiento comer- $\operatorname{cial}(\mathrm{CP})$}

Este concepto hace referencia a resultados de investigación generalmente surgidos de proyectos de investigación o de tesis desarrolladas en las IEIA que dan lugar a procesos o productos que pueden ser aprovechados de manera comercial y, eventualmente, cuentan incluso con protección de propiedad intelectual, pero no han sido llevados al mercado de manera formal. Cabe aclarar que, en este caso las IEIA cumplen también con el rol de OM, pues en ellas se generan conocimientos que son la base de esta modalidad.

A pesar de que estas actividades no son meramente emprendimientos, se incluyeron en el estudio pues los desarrolladores cuentan con reconocimiento en la comunidad universitaria, por lo que fueron considerablemente referidos y son una proporción importante $(36 \%$ de los 71 emprendedores-investigadores del padrón).

Los CP se refieren a variedades de granos, hortalizas, ornamentales, frutas, café, biofertilizantes, bioplaguicidas, alimentos funcionales, maquinaria agrícola y equipo agroindustrial, cuya producción se limita a pruebas a pequeña escala y en algunos casos a consultorías esporádicas y surgen por una visión hacia la academia, más que por una visión hacia el mercado.

En muchos casos los CP cuentan con patente, modelo de utilidad o título de obtentor, según sea el caso. Estos títulos avalan a la OM como propietario del conocimiento y a los investigadores como desarrolladores del producto. No obstante, existen CP que no cuentan con el título de propiedad intelectual correspondiente debido a que no se ha promovido por parte del investigador, ya que desean controlar su explotación comercial por su cuenta, porque no lo consideran necesario o porque hay cambios recurrentes en las oficinas de patentes de las OM que retrasan y dificultan los trámites.

En esta dinámica de generación de conocimiento, los investigadores disponen de la infraestructura de la OM, desde laboratorios, parcelas, invernaderos, reactivos, material y equipo de laboratorio, así como del personal técnico y administrativo. Cabe destacar que generalmente las investigaciones son realizadas a través de tesistas que colaboran con el investigador responsable.
Los CP se han generado en gran medida por estímulos económicos provenientes de la respectiva IEIA y por el Consejo Nacional de Ciencia y Tecnología (CONACYT) a través del Sistema Nacional de Investigadores (SNI). Estos resultados se difunden en eventos científicos y permite tanto a la OM como al investigador tener el reconocimiento académico, pero no existe una promoción comercial de los CP que permita generar valor económico. Es decir, para gestionar los recursos y hacer la investigación se presentan justificaciones económicas y apoyos de usuarios potenciales del conocimiento (productores, empresas, asociaciones), pero una vez concluida la misma, el cierre de los proyectos se hace con productos académicos (tesis, artículos, libros, patentes), dejando la promoción del uso del conocimiento como algo opcional y la valoración del uso del conocimiento generado como algo circunstancial.

Por ejemplo, el sector privado se ha interesado en adquirir las variedades generadas de hortalizas; sin embargo, estas iniciativas no se han concretado por cambios administrativos y trámites burocráticos que se tienen que llevar en las oficinas correspondientes dentro de las OM. Por consiguiente, los investigadores han optado por mantener el contacto por cuenta propia a productores independientes y a asociaciones para hacerles llegar el producto. Como se percibe, los clientes son los que promueven estos vínculos, más que el interés del propio investigador por concretar una actividad económica a través de la comercialización de los CP.

En este estudio se detectó que los investigadores desean mejorar sus ingresos, pero generalmente no tienen interés por convertirse en emprendedores alrededor del CP desarrollado. Al respecto, Sousa, Jorge Nassif, y Tozi (2014) mencionan que la introducción en el mercado del conocimiento científico requiere competencias que los investigadores normalmente no han desarrollado, por lo que posiblemente el desarrollador del CP no suele cumplir con el rol de emprendedor o para hacerlo necesite prepararse en áreas gerenciales.

En suma, los CP son resultados de investigación generalmente con protección legal a través de los cuales las IEIA se han posicionado con prestigio en sus áreas de investigación, pero no existe un esfuerzo deliberado por promover su actividad comercial a pesar de que muchas veces el mercado es el interesado por adquirir los productos o servicios.

\section{Empresas vinculadas (EV)}

Dentro de los emprendimientos se reconoce como EV a aquella entidad económica con fines de lucro involucrada en el sector agropecuario, que presenta una relación formal o informal con una IEIA, la cual le permite desarrollar redes de proveeduría, de conocimientos y de clientes; pero no centran su actividad económica en conocimientos generados en estas instituciones, por lo que son empresas cuyos productos y servicios no son tan innovadores. En esta modalidad existe un emprendedor como tal, que ha asumido riesgos y ha realizado inversiones.

Mediante las entrevistas se estudiaron cuatro empresas que cumplen con estas características. La relación que mantienen los emprendedores con la IEIA es como profesores investigadores, estudiantes, egresados o trabajadores (Cuadro 2). No obstante, desde el punto de vista de la 
EV la relación es informal y a conveniencia de los emprendedores. Por ello, se trata de una relación asimétrica donde los emprendedores aprovechan las externalidades positivas de la universidad para desarrollar una empresa. Cuadro 2. Caracterización de empresas vinculadas a IEIA

Cuadro 2: Caracterización de empresas vinculadas a IEIA

\begin{tabular}{|c|c|c|c|c|}
\hline \multirow{2}{*}{ Característica } & \multicolumn{4}{|c|}{ Empresas } \\
\hline & EV1 & EV2 & EV3 & EV4 \\
\hline Tipo de empresa & Servicios & Servicios & Productos y servicios & Productos y servicios \\
\hline Relación emprendedor-IEIA & Trabajador & Egresado & Estudiante & Profesor-investigador \\
\hline Financiamiento actual & Propio & Externo/propio & Propio & Externo/propio \\
\hline Uso del nombre de la IEIA & No usa & No usa & No usa & Uso indirecto \\
\hline Constitución legal & Sí & $\mathrm{Si}^{*}$ & Sí & $\mathrm{Si}^{\prime *}$ \\
\hline Número de empleados & 8 & 35 & 5 & 40 \\
\hline Involucramiento familiar & No & Sí & Sí & Sí \\
\hline Área de influencia & Nacional & Nacional & Regional & Nacional \\
\hline
\end{tabular}

${ }^{*} \mathrm{Al}$ menos ha formado dos figuras legales para operar.

Fuente: elaboración propia con información de entrevistas a emprendedores.

Las EV estudiadas surgen por interés del propio emprendedor, sin intervención de la IEIA y con el uso de capital propio; aunque en la operación actual, algunas de ellas hacen uso de recursos financieros externos. Todas son empresas legalmente constituidas, pero en el caso de la EV2 y la EV4 han formado al menos dos figuras legales para operar, esto debido al giro que tienen y porque están orientadas tanto a la venta de servicios como de productos.

Los giros comerciales de las EV van desde consultoría fitozoosanitaria, logística de comercialización internacional de productos agropecuarios, naturación urbana y distribución de insumos y productos agrícolas. Debido a esta característica y a su área de influencia, la cantidad de mano de obra contratada es muy variable.

El criterio "uso de nombre de la IEIA" hace referencia al hecho de que las empresas promocionan sus productos o servicios haciendo alusión al nombre de la IEIA (Cuadro 2), esto por el prestigio ligado a la institución. Se denomina de manera directa cuando el nombre de la institución aparece como tal en la etiqueta de los productos o al momento de comercializar los servicios, y es considerado de forma indirecta cuando la comercialización se realiza haciendo mención del cargo o relación que tiene el emprendedor dentro de la IEIA.

Por ejemplo, en su momento el emprendedor de la EV4 desarrolló actividades de capacitación y consultoría como profesor-investigador de la universidad; al observar el interés y potencial en la idea de negocio, fundó la empresa y actualmente distribuye productos y servicios, pero el mercado lo reconoce como académico de la IEIA. Ello sugiere que la relación que mantienen las EV con su IEIA deriva en redes de contactos para desarrollar una cartera de clientes.

Bajo este contexto de vinculación, las EV se ven beneficiadas también al disponer de personal especializado que puede formar parte de sus empleados. Además, el involucramiento laboral del emprendedor dentro de la IEIA le genera facilidades para atraer a tesistas, alumnos de servicio social o prácticas profesionales que realizan actividades de investigación, las cuales no necesariamente son para desarrollo de nuevos productos, sino para realizar pruebas a los productos que comercializan o involucrarlos en los procesos laborales de rutina. En este sentido, la IEIA se ve beneficiada de manera directa, pues a través de la vinculación de alumnos con las EV se perfeccionan las competencias de sus estudiantes, se cumplen requisitos de graduación, impactando en la eficiencia terminal de las IEIA y en un sentido más amplio, las EV son fuente de empleo para sus egresados.

En resumen, en las EV se refleja un compromiso del emprendedor por establecer una empresa y, a pesar de que los productos o servicios que comercializan las EV no son tan innovadores, estas empresas mantienen relación con una IEIA, la cual les permite atraer a capital humano especializado, así como desarrollar redes de proveeduría, de conocimientos y de clientes que beneficia tanto a la EV como a la IEIA.

\section{Empresas de base tecnológica (EBT)}

Finalmente, se considera como EBT a una entidad con fines de lucro que organiza recursos financieros, humanos y tecnológicos con el fin de comercializar conocimientos generados en una IEIA a través de la venta de servicios o productos. Por lo tanto, está establecida en torno a uno o varios desarrollos científicos originados en una institución de investigación, de manera tal que existe un proceso de transferencia del conocimiento de forma intermitente. Al igual que sucede en los $\mathrm{CP}$, las IEIA cumplen también con el rol de OM para el caso de las EBT. En esta categoría se analizaron nueve empresas que cumplen con estas características y para fines de esta investigación las EBT consideradas son empresas que se desenvuelven en el sector agropecuario. 
Al igual de lo que reportan De la O Barroso-González, Jiménez-García y Pérez-González (2014), la mayor parte de las EBT analizadas, tienen como fundador a emprendedores que tienen o tuvieron relación con la OM como profesores-investigadores y son menos las empresas fundadas por egresados. También se identificó que, para todos los casos, las EBT que se dedican a la venta de productos, lo hacen también con servicios asociados, es decir, no solo venden los productos, sino que también ofrecen asesorías, capacitaciones o instalación vinculados a los productos (Cuadro 3).Cuadro 3.

Cuadro 3: Caracterización de empresas de base tecnológica asociadas a IEIA

\begin{tabular}{|c|c|c|c|c|c|c|}
\hline \multirow{4}{*}{ Característica } & \multicolumn{6}{|c|}{ Empresa } \\
\hline & \multirow{3}{*}{ EBT1 } & \multirow{3}{*}{ EBT2 } & \multirow{3}{*}{ EBT3* $^{*}$} & \multirow{3}{*}{ EBT4 } & \multirow{3}{*}{$\begin{array}{l}\text { EBT5 }^{*} \\
\text { EBT6 } \\
\text { EBT7 }\end{array}$} & \multirow{3}{*}{$\begin{array}{l}\text { EBT8 } \\
\text { EBT9* }^{*}\end{array}$} \\
\hline & & & & & & \\
\hline & & & & & & \\
\hline $\begin{array}{l}\text { Conocimiento } \\
\text { transferido }\end{array}$ & $\begin{array}{l}\text { Intangible } \\
\text { (servicios) }\end{array}$ & $\begin{array}{l}\text { Tangible e intangible } \\
\text { (servicios y productos) }\end{array}$ & $\begin{array}{l}\text { Tangible e intangible } \\
\text { (servicios y productos) }\end{array}$ & $\begin{array}{l}\text { Tangible e intangible } \\
\text { (servicios y productos) }\end{array}$ & $\begin{array}{l}\text { Tangible e intangible } \\
\text { (servicios y productos) }\end{array}$ & $\begin{array}{l}\text { Tangible e intangible } \\
\text { (servicios y productos) }\end{array}$ \\
\hline $\begin{array}{l}\text { Relación emprende- } \\
\text { dor-OM }\end{array}$ & $\begin{array}{l}\text { Profesor- } \\
\text { investigador }\end{array}$ & Egresado & $\begin{array}{l}\text { Profesor-investi- } \\
\text { gador }\end{array}$ & Profesor-investigador & Profesor-investigador & $\begin{array}{l}\text { Exprofesor-investi- } \\
\text { gador }\end{array}$ \\
\hline $\begin{array}{l}\text { Tipo de relación } \\
\text { emprendedor-IEIA }\end{array}$ & Formal & Ninguna & Formal & Formal & Formal & Ninguna \\
\hline Constitución legal & No & Sí & Sí & No & Sí & Sí \\
\hline $\begin{array}{l}\text { Involucramiento } \\
\text { familiar }\end{array}$ & No & Sí & Sí & No & Sí & Sí \\
\hline Área de influencia & Internacional & Regional & Nacional & Regional & Regional & Regional \\
\hline
\end{tabular}

${ }^{\star} \mathrm{Al}$ menos ha formado dos figuras legales para operar.

Fuente: elaboración propia con información de entrevistas a emprendedores.

Las EBT participan en una amplia gama de actividades que incluyen capacitación y asesoría en reproducción animal, energías sustentables, producción de bio fertilizantes, paquetes tecnológicos en maguey mezcal, nutrición y suplementación animal, así como naturación forestal y urbana.

$\mathrm{Al}$ igual que las EV, la mayoría de las EBT son empresas legalmente constituidas y en tres de los siete casos de empresas orientadas a servicios y productos, han constituido al menos dos figuras legales para comercializar ambos tipos de mercancías.

En general, las EBT se localizan geográficamente cerca de la OM, sin embargo, su área de influencia va desde lo regional a lo nacional y para el caso de la EBT1, dedicada a la capacitación y consultoría en reproducción animal, su área de influencia llega a EE. UU. y Centroamérica (Cuadro 4).

Resulta importante mencionar que en esta modalidad los emprendedores tienden a usar más el nombre de la $\mathrm{OM}$ en el proceso de comercialización, comparados con la modalidad de EV. Situación que puede explicarse a que los emprendedores quieren reflejar efectividad de sus productos y procesos a través de esta asociación.
Debido a la relación que tiene el emprendedor con la OM, el acceso a los estudiantes y egresados especializados en el sector agropecuario resulta ser un recurso valioso para el funcionamiento de estas empresas. En este sentido, los estudiantes pueden hacer trabajos de tesis mediante los cuales se desarrollan los conocimientos que serán promovidos por la EBT, posteriormente los alumnos pueden convertirse en empleados de estas empresas; siendo así una fuente de empleo para egresados de las OM.

Las investigaciones se llevan a cabo a través de proyectos o tesis en donde los emprendedores fungen como responsables y tienen acceso a infraestructura de la OM, como laboratorios, equipos especializados, reactivos, tesistas, personal técnico y administrativo, entre otros. Mientras que las investigaciones que realizan las EV están orientadas hacia la prueba de productos o procesos ya generados, las investigaciones de las EBT están orientadas a generar nuevos productos o procesos que tienen una visión hacia el mercado.

Así, el financiamiento de la generación de conocimientos, y en su caso, el trámite de títulos de propiedad fue por parte de la OM; sin embargo, la comercialización de estos resultados de investigaciones se hace de forma no regulada y sin vinculación de cooperación entre ambas entidades. 
$\mathrm{Al}$ igual que sucede con las EV, la relación que mantiene el emprendedor con la OM resulta favorable pues se dispone de contactos de una red de conocimiento de diferentes áreas dentro de la universidad, así como del posible desarrollo de la red comercial de la empresa debido al reconocimiento del emprendedor.

No obstante, las OM se dedican mayoritariamente a actividades de docencia e investigación, sin tomar un rol de generación o acompañamiento de empresas y por su parte, los emprendedores mantienen el vínculo académico con las OM, pero constituyen las EBT de manera autónoma, por lo que los beneficios de estos vínculos son limitados para ambos actores comparados con varios estudios (Aportela Rodríguez \& Gallego Gómez, 2015; Martínez Román \& Gómez Miranda, 2014; Mustar, 1997; Steffensen et al., 2000); precisamente por trabajar de manera independiente.

Específicamente, el COLPOS ha desarrollado normatividad para regular sus relaciones con empresas, incluidas las EBT, pero los emprendedores aluden que los trámites a realizar impiden que sea un proceso ágil, por lo que las empresas prefieren no atravesar por ello y generan empresas independientes a la OM.

Por otro lado, no existe oferta de financiamiento por parte de las OM, como en varios países se ha establecido, por lo que en la creación y desarrollo de estas EBT se ve involucrado el capital propio del emprendedor y en algunos casos de financiamiento externo.

Otra de las situaciones a considerar es que el personal directivo de las EBT suele tener una formación especializada en aspectos técnicos que forman parte central de los procesos, pero no tienen el mismo grado de formación en aspectos gerenciales y de dirección de empresas. Con relación a lo anterior, Abreu et al. (2016), consideran que el papel de la universidad debe extenderse a actividades que promueven el pensamiento empresarial, los valores y las instituciones, conocido como "capital emprendedor".

En resumen, las EBT de este estudio son empresas formalmente constituidas que se han creado alrededor un desarrollo científico originado en la IEIA con el compromiso solo de los fundadores; pero, dado el rol del emprendedor dentro de la institución, se generan beneficios sin la intervención formal de la IEIA que han sido poco valorados por los emprendedores y que les ha permitido generar prestigio en sus productos o procesos en el mercado.

\section{El ecosistema emprendedor en las IEIA}

Los conocimientos que ocasionalmente llegan al mercado (CP) y los emprendimientos formales (EV y EBT) anteriormente descritos constituyen el actor central del ecosistema emprendedor analizados.

Por otra parte, la normatividad interna del COLPOS establece criterios para la creación y desarrollo de empresas, pero han sido poco eficaces para el caso de EV y casi nada en el caso de EBT. Mientras que, en la UACh, no existe normatividad explícita para regular la creación y vinculación de empresas; por lo que las EV y EBT derivadas de estas instituciones se han creado de manera independiente a la universidad y por interés de los propios emprendedores. Bajo este contexto, se puede ver que en las IEIA estudiadas no existe una política formal de apoyo al emprendimiento de la cual se derive en mecanismos para crear y desarrollar empresas en el que los actores involucrados tengan objetivos operativos que cumplir, por lo que no han sido capaces de ser un agente clave en el proceso emprendedor asociado a ellas (Cuadro 4).

Cuadro 4: Caracterización de las IEIA

\begin{tabular}{|c|c|c|}
\hline IEIA & COLPOS & UACh \\
\hline Funciones específicas & Educación, investigación y vinculación & Educación, investigación, servicio y difusión de la cultura \\
\hline Áreas de investigación & $\begin{array}{l}\text { Agroecología y sustentabilidad, edafología, fitosanidad, } \\
\text { ciencias forestales, hidrociencias, recursos genéticos y pro- } \\
\text { ductividad, socioeconomía, estadística e } \\
\text { informática }\end{array}$ & $\begin{array}{l}\text { Agroecología, ciencias forestales, ciencias económico-administra- } \\
\text { tivas, fitotecnia, irrigación, agroindustria, mecánica agrícola, para- } \\
\text { sitología, sociología rural, suelos y zootecnia }\end{array}$ \\
\hline Programas educativos & 16 maestrías y 11 doctorados & $\begin{array}{l}\text { Preparatoria agrícola, propedéutico, } 27 \text { licenciaturas, } 16 \text { maestrías } \\
\text { y } 12 \text { doctorados }\end{array}$ \\
\hline Número de investigadores & 435 (283 pertenecientes al SNI) & 924 (159 pertenecientes al SNI) \\
\hline $\begin{array}{l}\text { Oficinas de transferencia de } \\
\text { tecnología }\end{array}$ & Establecidas & No oficialmente \\
\hline Normatividad interna & Lineamientos establecidos, pero poco implementados & No explícitamente \\
\hline Mecanismos implementados & Iniciativas por parte de investigadores & Iniciativas por parte de investigadores \\
\hline
\end{tabular}

Fuente: elaboración propia con información de revisión documental y de entrevistas.

Las actividades realizadas por la universidad juegan un papel importante en la creación y desarrollo de EBT. Este fenómeno ya no puede limitarse al contexto de las instituciones de investigación y debe trascender hasta el diseño e implementación de políticas universitarias de inversión de capital, en la creación de la oficina de patentes y mantener una participación de los inventores en las regalías para aumentar la actividad empresarial (Abreu et al., 2016). Lo anterior está respaldado por la evidencia de estudios de caso sobre universidades menos intensivas 
en investigación que se convierten en facilitadores cada vez más importantes y proactivas de innovación y emprendimiento (Abreu et al., 2016; Di Gregorio \& Shane, 2003; Pirnay, Surlemont, \& Nlemvo, 2003).

Al respecto, la Ley de Ciencia y Tecnología en México (LCyT), a través de CONACYT, juega un papel importante en este ecosistema generando capital humano especializado proveniente de las IEIA a través de becas para alumnos de posgrado. También, derivado de esta ley se han constituido dentro de las IEIA centros públicos de investigación y laboratorios certificados que estimulan las actividades de investigación. Así mismo, se incentiva económicamente a los investigadores a producir conocimientos científicos y tecnológicos a través del SNI.

En lo que se refiere al capital humano, el COLPOS se ha posicionado como una institución pública de educación de posgrado e investigación, mientras que la oferta formativa de la UACh incluye educación a nivel de bachillerato, licenciatura y posgrado (Cuadro 4). En este sentido, como ya se ha mencionado, las EV y las EBT implican una fuente de empleo para los egresados de las IEIA.

Por otro lado, el tema del financiamiento se ha visualizado como un punto crítico al momento de generar un emprendimiento asociado a una IEIA (Rodeiro Pazos et al., 2010). La generación de CP se realiza a través de las propias instituciones dadas las funciones que tienen los investigadores.
Cabe recordar que, el desarrollo de investigaciones que realizan las EV y EBT muchas veces se realiza con infraestructura, materiales y equipo de las IEIA a través de los proyectos de investigación y tesis. Mientras que, para las inversiones de puesta en marcha y operación en estas modalidades, son costeadas por el propio emprendedor y en algunos casos por fuentes externas, principalmente por medio de la banca privada.

Con respecto al mercado, la mayoría de EV y EBT tienen influencia regional; pero hay casos, principalmente en la venta de servicios, en donde éstos son llevados al territorio nacional y en uno de los casos al ámbito internacional. De igual forma, algunos gobiernos estatales son clientes principales de algunas de estas empresas.

Lo anteriormente expuesto refleja que en el ecosistema emprendedor analizado interactúan seis actores: las políticas públicas, las IEIA, el capital humano, los emprendimientos, el financiamiento y el mercado. Así, el CONACYT estimula por diversas vías a las IEIA para generar conocimientos científicos y tecnológicos, convirtiéndose en $\mathrm{OM}$; de igual manera, forma capital humano que es empleado por empresas, ya sea vinculadas o de base tecnológica para generar y comercializar productos o servicios al mercado regional y nacional, principalmente (Figura 2).

Figura 2: Ecosistema emprendedor universitario de las IEIA



Fuente: Elaboración propia con base en (Audretsch \& Belitski, 2017; Isenberg, 2010; Nicotra et al., 2018; Znagui \& Rahmouni, 2019) 
Es conveniente destacar que los roles de los actores no son específicos y claros para promover el emprendimiento asociado a las IEIA analizadas, pues tanto las políticas públicas como las universidades tienen una fuerte orientación hacia la docencia e investigación de manera que la generación de resultados de investigaciones, así como de capital humano son altamente reconocidos. No obstante, es casi inexistente la orientación hacia el emprendimiento, de ahí que sean los emprendedores los que promuevan las iniciativas empresariales. Es decir, en el ecosistema analizado la productividad científica y capital humano es promovida por las IEIA y las políticas públicas, mientras que las actividades de emprendimiento quedan a cargo prácticamente de los emprendedores.

\section{Retos y oportunidades para el ecosistema emprendedor en las IEIA}

Diversas universidades han integrado ecosistemas de emprendimiento dentro de su organización (Pérez-Zavala, Molina Sanchez, Schmitt, \& López Salazar, 2019); sin embargo, el proceso de comercialización de la investigación es complejo debido a una diferencia entre la cultura y normativa de la universidad y la de los negocios, por lo que la transición debe gestionarse a fin de que estas diferencias no se conviertan en una barrera para la transferencia de conocimiento (Ellwood, Williams, \& Egan, 2020; Markham, Ward, Aiman-Smith, \& Kingon, 2010).
Como punto de partida, las IEIA involucradas en este estudio pueden desarrollar una estrategia para primero identificar y luego promover el emprendimiento. Este proceso requiere la planeación e intervención coordinada de diferentes actores, entre ellos, el investigador, el emprendedor, la IEIA y el sector hacia el cual se dirigirán los productos y servicios.

Dado que en los CP los investigadores no tienen experiencia como empresarios y están más interesados en la enseñanza, la publicación científica y la ciencia abierta, se sugiere impulsar su comercialización a través del modelo de emprendedor sustituto (Würmseher, 2017), vinculando a estos investigadores con emprendedores externos.

Para esto, las IEIA tienen que reconocer y evaluar el potencial técnico y comercial de los CP a fin de avalarlos y promocionarlos para incrementar el interés de empresarios externos por comercializarlos. Sería conveniente considerar en este proceso a las EV como emprendedor sustituto para crear alianzas estratégicas como resultado de su experiencia previa en el mercado. En el caso de las EBT, se recomienda implementar el modelo inventor emprendedor (Würmseher, 2017) a través del desarrollo de capacidades gerenciales de los emprendedores (Figura 3).

Figura 3: Propuesta de acción para incentivar los emprendimientos en las IEIA

\begin{tabular}{|c|c|c|}
\hline $\begin{array}{l}\text { Orienta la investigación que realiza } \\
\text { hacia las necesidades del mercado. } \\
\text { Tiene interés por emprender. }\end{array}$ & $\begin{array}{l}\text { Formar capacidades gerenciales } \\
\text { en el emprendedor }\end{array}$ & $\begin{array}{c}\text { Empresas de base tecnológica (EBT) } \\
\text { El investigador comercializa sus } \\
\text { investigaciones por cuenta propia a } \\
\text { través de una EBT fundada por él } \\
\text { mismo } \\
\text { (Modelo de inventor emprendedor) }\end{array}$ \\
\hline $\begin{array}{l}\text { Conocimientos con potencial de } \\
\text { aprovechamiento comercial (CP) } \\
\text { El investigador enfoca sus actividades a } \\
\text { objetivos académicos. Sin aspiración de } \\
\text { participar en un proyecto de inversión }\end{array}$ & $\begin{array}{l}\text { - Promoción y evaluación de CP } \\
\text { - Vincular al investigador y al } \\
\text { emprendedor desde etapas tempranas } \\
\text { para empatar objetivos de } \\
\text { investigación }\end{array}$ & $\begin{array}{l}\text { Empresas externas (EE) } \\
\text { La EE (actor externo a la investigación) } \\
\text { se interesa por comercializar los CP. } \\
\text { Puede ser EV. } \\
\text { (modelo de emprendedor sustituto) }\end{array}$ \\
\hline \multicolumn{3}{|c|}{$\begin{array}{l}\text { - Asesoría legal y financiera para los emprendedores. } \\
\text { - Fomentar el emprendimiento a través de cursos con valor curricular para alumr } \\
\text { - Ser aval técnico en modelos de financiamiento como el crowfounding. } \\
\text { - Incentivar la movilidad de los investigadores hacia el sector productivo para or } \\
\text { - Gestionar la interacción entre emprendedores universitarios generando redes d }\end{array}$} \\
\hline
\end{tabular}

Fuente: elaboración propia con base en Würmseher (2017). 
Resulta importante considerar que los emprendimientos académicos son heterogéneos, por lo que las IEIA interesadas en implementar estas acciones deben asegurarse de que los actores involucrados tengan una comprensión clara de los modelos emprendedores existentes, pues para cada caso se debe evaluar cuál es la mejor vía de aprovechamiento productivo (Clarysse, Wright, Lockett, Van de Velde, \& Vohora, 2005; Würmseher, 2017).

Además de las acciones específicas para cada modelo emprendedor, se considera pertinente implementar líneas estratégicas transversales para fomentar el emprendimiento asociado a las IEIA. Una de ellas es que éstas proporcionen asesoría legal y financiera para los tres tipos de modalidades empresariales (CP, EV y EBT) con la finalidad de que los emprendedores puedan ser orientados en estos aspectos, tomando en cuenta que es una de las áreas en donde normalmente no tienen experiencia.

Dado que, en el medio académico de las IEIA, en general se carece de capacidades gerenciales, las instituciones podrían intervenir en la formación de capital emprendedor a través de cursos con validez curricular y programas orientados al emprendimiento, administración y dirección. Estos cursos pueden impartirse por mentores en las IEIA con experiencia en el medio empresarial y orientarlos hacia conocimientos y habilidades prácticas más que teóricas, con la finalidad de desarrollar habilidades directivas y generar un impacto en su propensión a convertirse en empresarios (Anderseck, 2004; Muscio \& Ramaciotti, 2019).

Por otro lado, el tema de financiamiento es una de las dificultades más relevantes debido a que las EV y EBT son pequeñas y relativamente nuevas (Ndonzuau et al., 2002). Bajo esta premisa, posiblemente las IEIA no puedan involucrarse como un ente financiador, pero sí pueden participar como aval técnico en modelos de financiamiento como el crowfounding o fondos concursables.

También se propone incentivar la vinculación y movilidad de los investigadores con los sectores industriales a fin de generar investigaciones orientadas hacia el mercado, así como catalizar la interacción entre emprendedores universitarios para generar redes de conocimiento que les permita socializar e intercambiar experiencias relacionadas con su labor como emprendedores y con temas de índole tecnológico.

En esta etapa de despegue se considera pertinente implementar acciones en las oficinas ya existentes de las IEIA; más que crear nuevas estructuras, a fin de desarrollar y probar la normatividad institucional en donde se establezcan los roles que juegan los diferentes actores, así como los planes de vinculación.

Esta intervención debe resultar de fácil implementación, tanto para las propias IEIA como para las empresas, pues se desea impulsar emprendimientos productivos y no generar recursos propios para las IEIA, que es donde las normatividades actuales enfatizan y que paradójicamente obstaculizan su aplicación. Por ello, es de particular relevancia que el criterio de desempeño de estas acciones esté asociado a los emprendimientos productivos generados en el marco del programa.
Posiblemente, cuando estas acciones sean afianzadas por los actores involucrados a través de resultados positivos, las estructuras existentes puedan formalizarse como incubadoras de empresas y la IEIA se permita tener algún tipo de retribución derivado de estos modelos.

\section{Conclusiones y recomendaciones}

Las IEIA generan conocimientos y desarrollos tecnológicos con posibilidad de utilidad comercial. Sin embargo, la gran mayoría, permanecen como conocimientos con potencial, ya que no son llevados al mercado de manera formal. Existen también emprendimientos que están asociados a las IEIA pero que en su actividad no incorporan conocimientos generados en la universidad y que corresponden a empresas más o menos convencionales. En este universo, los emprendimientos basados en los conocimientos generados por las IEIA son minoritarios y a pesar de su dependencia, al menos en su origen, suelen mantener vínculos informales con las IEIA, pero las evidencias de buenas prácticas para su creación y consolidación no se documentan.

Lo anterior significa que el ecosistema emprendedor analizado está muy poco desarrollado, pues los logros son más bien circunstanciales y no son resultado de una estrategia bien definida para lograr una aplicación rápida del conocimiento generado en las IEIA. Así, existen iniciativas empresariales que son dispersas y que generalmente son impulsadas solo por los emprendedores y no tienen un impacto relevante al nivel institucional.

La falta de sistematización de las experiencias exitosas en materia de EBT no ha permitido entender el ciclo de vida de estas empresas y el papel que deben jugar los diferentes actores en cada una de sus etapas por lo que existe una escasa interacción entre los actores mermando la capacidad de crear un ecosistema emprendedor universitario funcional. De hecho, al interior de las IEIA se tiende a sobrevalorar la aportación del conocimiento generado, en detrimento de los otros dos factores limitantes para el desarrollo de una EBT: la dotación de habilidades directivas y empresariales y el acceso a financiamiento.

Lo anterior, requiere cambios en la cultura y estructuras de las IEIA, así como cambios en las políticas públicas de incentivos a los investigadores, donde también se premie la vinculación con el sector productivo, al mismo nivel que la publicación de artículos científicos. En el corto plazo, se cree más factible reconocer y evaluar los CP a fin de determinar su potencial económico y comercial para promocionarlos; generar habilidades directivas en los emprendedores; proporcionar asesoría legal y financiera; y empatar los objetivos de investigación en las IEIA con el mercado. Para tal efecto, se considera la restructuración de algunas funciones en áreas ya existentes dentro de las IEIA, para que se conciban como un ente de fomento al capital emprendedor y no solo como divulgadores de conocimiento o gestores de servicios universitarios. 


\section{Referencias}

Abreu, M., Demirel, P., Grinevich, V., \& Karataş-Özkan, M. (2016). Entrepreneurial practices in research-intensive and teaching-led universities. Small Business Economics, 47(3), 695-717. https://doi. org/10.1007/s11187-016-9754-5

América Economía. (2021). Las mejores universidades de México 2020. Retrieved January 20, 2005, from https://mba.americaeconomia.com/articulos/notas/las-mejores-universidades-de-mexico-2020

Anderseck, K. (2004). Institutional and academic entrepreneurship: implications for university governance and management. Higher Education in Europe, 29(2), 193-200. https://doi. org/10.1080/0379772042000234820

Aportela Rodríguez, I. M., \& Gallego Gómez, C. (2015). La información como recurso estratégico en las empresas de base tecnológica. Revista General de Información Y Documentación, 25(2), 265285. https://doi.org/http://dx.doi.org/10.5209/rev_RGID.2015.v25. n 2.51238

Audretsch, D. B., \& Belitski, M. (2017). Entrepreneurial ecosystems in cities: establishing the framework conditions. Journal of Technology Transfer, 42(5), 1030-1051. https://doi.org/10.1007/s10961-0169473-8

Audretsch, D. B., Cunningham, J. A., Kuratko, D. F., Lehmann, E. E., \& Menter, M. (2019). Entrepreneurial ecosystems: economic, technological, and societal impacts. Journal of Technology Transfer, 44(2), 313-325. https://doi.org/10.1007/s10961-018-9690-4

Cáceres Carrasco, F. R., \& Aceytuno, M. T. (2015). Academic spin-offs incubation strategies: The case of the Andalusian region. Cuadernos de Gestion, 15(2), 113-142. https://doi.org/10.5295/cdg.140479ma

Cai, L., Peng, X., \& Wang, L. (2018). The characteristics and influencing factors of entrepreneurial behaviour: The case of new stateowned firms in the new energy automobile industry in an emerging economy. Technological Forecasting and Social Change, 135(235), 112-120. https://doi.org/10.1016/j.techfore.2018.04.014

Carayannis, E. G., Rogers, E. M., Kurihara, K., \& Allbritton, M. M. (1998). High-technology spin-offs from government R\&D laboratories and research universities. Technovation, 18(1), 1-11. https://doi. org/10.1016/S0166-4972(97)00101-6

Clark, B. R. (1998). Creating entrepreneurial universities. Organizational pathways of transformation. Paris, Francia: IAU Press.

Clarysse, B., Wright, M., Lockett, A., Van de Velde, E., \& Vohora, A. (2005). Spinning out new ventures: A typology of incubation strategies from European research institutions. Journal of Business Venturing, 20(2), 183-216. https://doi.org/10.1016/j.jbusvent.2003.12.004
De la O Barroso-González, M., Jiménez-García, M., \& Pérez-González, M. del C. (2014). Incidencia de diferentes sistemas territoriales de innovación en la creación de Empresas de Base Tecnológica (EBTs). Una aplicación al caso andaluz. Journal Globalization, Competitiveness and Governability, 8(3), 62-82. https://doi.org/10.3232/ GCG.2014.V8.N3.04

Di Gregorio, D., \& Shane, S. (2003). Why do some universities generate more start-ups than others? Research Policy, 32(2 SPEC.), 209227. https://doi.org/10.1016/S0048-7333(02)00097-5

Ellwood, P., Williams, C., \& Egan, J. (2020). Crossing the valley of death: Five underlying innovation processes. Technovation, (August 2018), 102162. https://doi.org/10.1016/j.technovation.2020.102162

Etzkowitz, H., Webster, A., Gebhardt, C., \& Cantisano Terra, B. R. (2000). The future of the university and the university of the future: evolution of ivory tower to entrepreneurial paradigm. Research Policy, $29,313-330$

Guerrero, M., Urbano, D., Fayolle, A., Klofsten, M., \& Mian, S. (2016). Entrepreneurial universities: emerging models in the new social and economic landscape. Small Business Economics, 47(3), 551-563. https://doi.org/10.1007/s11187-016-9755-4

Hacker, S. R., Klaesson, J., Pettersson, L., \& Sjölander, P. (2013). Urban growth: Co-evolution of producer services and other sectors. In J. Klaesson, B. Johansson, \& C. Karlsson (Eds.), Metropolitan Regions: Knowledge Infrastructures of the Global Economy (pp. 117-139). Berlin, Heidelberg: Springer. https://doi.org/10.1007/978-3-642-32141-2

Hisrich, R. D., Ge, B., Gao, H., \& Sheng, F. (2016). Frontiers on Research of Innovation and Entrepreneurship: Review of the Keynotes of "The 2015 International Conference on Innovation and Entrepreneurship." Journal of Industrial Integration and Management, 1(2), 1650006. https://doi.org/10.1142/s2424862216500068

Huang-Saad, A., Fay, J., \& Sheridan, L. (2017). Closing the divide: accelerating technology commercialization by catalyzing the university entrepreneurial ecosystem with I-Corps ${ }^{\mathrm{TM}}$. Journal of Technology Transfer, 42(6), 1466-1486. https://doi.org/10.1007/s10961-016-9531-2

Isenberg, D. J. (2010). The big idea: How to start an entrepreneurial revolution. Harvard Business Review, 88(6), 1-11.

Kalar, B., \& Antoncic, B. (2015). The entrepreneurial university, academic activities and technology and knowledge transfer in four European countries. Technovation, 36, 1-11. https://doi.org/10.1016/j. technovation.2014.11.002

Klofsten, M., \& Jones-Evans, D. (2000). Comparing Academic Entrepreneurship in Europe - The Case of Sweden and Ireland. Small Business Economics, 14(4), 299-309. https://doi. org/10.1023/a:1008184601282 
Lahikainen, K., Kolhinen, J., Ruskovaara, E., \& Pihkala, T. (2019). Challenges to the development of an entrepreneurial university ecosystem: The case of a Finnish university campus. Industry and Higher Education, 33(2), 96-107. https://doi.org/10.1177/0950422218815806

Markham, S. K., Ward, S. J., Aiman-Smith, L., \& Kingon, A. I. (2010). The valley of death as context for role theory in product innovation. Journal of Product Innovation Management, 27(3), 402-417. https:// doi.org/10.1111/j.1540-5885.2010.00724.x

Martínez Román, I., \& Gómez Miranda, M. E. (2014). Las spin-off universitarias en Andalucía: caracterización económico-financiera. Revista de Estudios Regionales, 99, 75-101.

Mason, C., \& Brown, R. (2014). Entrepreneurial ecosystems and growth oriented entreprenueurship. Netherlands: OECD. https://doi. org/10.4337/9781785364624

Meredith, G. G., Nelson, R. E., \& Neck, P. A. (1986). Lo que todo pequeño empresario debe saber. Ginebra, Suiza: Oficina Internacional del Trabajo.

Merritt, H. (2012). Las empresas mexicanas de base tecnológica y sus capacidades de innovación: una propuesta metodológicca. Trayectorias, 33, 27-50.

Miller, K., Mcadam, M., \& Mcadam, R. (2014). The changing university business model: A stakeholder perspective. $R$ and D Management, 44(3), 265-287. https://doi.org/10.1111/radm.12064

Muscio, A., \& Ramaciotti, L. (2019). How does academia influence Ph.D. entrepreneurship? New insights on the entrepreneurial university. Technovation, 82-83(January), 16-24. https://doi.org/10.1016/j. technovation.2019.02.003

Mustar, P. (1997). Spin-off enterprises. How french academics create hi-tech companies: the conditions for success or failure. Science and Public Policy, 24(I), 37-43.

Ndonzuau, F. N., Pirnay, F., \& Surlemont, B. (2002). A stage model of academic spin-off creation. Technovation, 22(5), 281-289. https://doi. org/10.1016/S0166-4972(01)00019-0

Nicotra, M., Romano, M., Del Giudice, M., \& Schillaci, C. E. (2018). The causal relation between entrepreneurial ecosystem and productive entrepreneurship: a measurement framework. Journal of Technology Transfer, 43(3), 640-673. https://doi.org/10.1007/s10961-017-9628-2

OCDE. (2012). Evaluación de la OECD del sector de las nuevas empresas basadas en el conocimiento. México. OCDE.

Pérez-Zavala, M. G., Molina Sanchez, R., Schmitt, C., \& López Salazar, A. (2019). Emprendimiento en dos universidades públicas: Universidad de Guanajuato, México y Université de Lorraine, France. Projectics / Proyéctica / Projectique, n²3, 99-116. https://doi.org/10.3917/ proj.023.0099
Pirnay, F., Surlemont, B., \& Nlemvo, F. (2003). Toward a typology of university spin-offs. Small Business Economics, 21, 355-369. https:// doi.org/10.1023/A:1026167105153

Prencipe, A., Corsi, C., Rodríguez-Gulías, M. J., Fernández-López, S., \& Rodeiro-Pazos, D. (2020). Influence of the regional entrepreneurial ecosystem and its knowledge spillovers in developing successful university spin-offs. Socio-Economic Planning Sciences. https://doi. org/10.1016/j.seps.2020.100814

QS Quacquarelli Symonds. (2021). World University Rankings 2022. Retrieved January 20, 2006, from https://www.topuniversities.com/ university-rankings/world-university-rankings/2022

Roberts, E. B., \& Malone, D. E. (1996). Policies and structures for spinning off new companies from research and development organizations. $R$ and D Management, 26(1), 17-48. https://doi. org/10.1111/j.1467-9310.1996.tb00927.x

Rodeiro Pazos, D., Fernández López, S., Rodríguez Sandiás, A., \& Otero González, L. (2010). Obstáculos para as spin-offs universitarias en España e en Galicia. Revista Galega de Economía, 19(1), 175-198. https://doi.org/10.1016/0883-9026(88)90008-0

Schillo, R. S. (2018). Research-based spin-offs as agents in the entrepreneurial ecosystem. Journal of Technology Transfer, 43(1), 222-239. https://doi.org/10.1007/s10961-016-9484-5

Shannon, C. E. (1948). A mathematical theory of communication. The Bell System Technical Journal, 27(4), 623-656. https://doi. org/10.1002/j.1538-7305.1948.tb00917.x

Sharma, P., \& Chrisman, J. J. (1999). Toward a reconciliation of the definitional issues of corporate entreprenuership. Entrepreneurship Theory and Practice, 11-28. Retrieved from http://web.ebscohost. $\mathrm{com} / \mathrm{ehost} / \mathrm{pdfviewer} / \mathrm{pdfviewer}$ ? sid $=66602 \mathrm{ba} 0-\mathrm{d} 878-4208-917 \mathrm{c}$ a02cccc0c5fb@sessionmgr115\&vid=2\&hid=126

Shearman, C., \& Burrell, G. (1988). New technology based firms and the emergence of new industries: some employment implications. New Technology, Work and Employment, 3(2), 87-99. https://doi. org/10.1111/j.1468-005X.1988.tb00092.x

Siegel, D. S., Waldman, D. A., Atwater, L. E., \& Link, A. N. (2003). Commercial knowledge transfers from universities to firms: Improving the effectiveness of university-industry collaboration. Journal of High Technology Management Research, 14(1), 111-133. https://doi. org/10.1016/S1047-8310(03)00007-5

Sousa, V. J., Jorge Nassif, M. V., \& Tozi, L. A. (2014). A cooperação universidade-empresa, as redes sociais e a difusão do conhecimento. Revista Brasileira de Gestão E Desenvolvimento Regional, 11(3), 178-204.

Spigel, B., \& Harrison, R. (2017). Towards a process theory of entrepreneurial ecosystems. Strategic Entrepreneurship Journal, 12, 151168. https://doi.org/10.1111/sej.1268 
Steffensen, M., Rogers, E. M., \& Speakman, K. (2000). Spin-offs from research centers at a research university. Journal of Business Venturing, 15(1), 93-111. https://doi.org/10.1016/S0883-9026(98)00006-8

Universidad Nacional Autónoma de México. (2020). Estudio comparativo de universidades mexicanas. Retrieved from http://www.execum.unam.mx/\#

Vitón, R., Castillo, A., \& Lopes Teixeira, T. (2019). Mapa de la innovación AG-TECH en América Latina y el Caribe. https://doi.org/http:// dx.doi.org/10.18235/0001788
Warf, B., \& Vincent, P. (2007). Religious diversity across the globe: A geographic exploration. Social and Cultural Geography, 8(4), $597-$ 613. https://doi.org/10.1080/14649360701529857

Würmseher, M. (2017). To each his own: Matching different entrepreneurial models to the academic scientist's individual needs. Technovation, 59(March), 1-17. https://doi.org/10.1016/j.technovation.2016.10.002

Znagui, Z., \& Rahmouni, B. (2019). What ecosystem model to support the creation of social innovation technopoles? Procedia Computer Science, 158, 877-884. https://doi.org/10.1016/j.procs.2019.09.126 